\title{
Desalination by Forward Osmosis: Identifying Performance Limiting Parameters through Module-Scale Modeling
}

\author{
Journal of Membrane Science
}

Submitted: November 24, 2014

Revised: February 26, 2015

\author{
Akshay Deshmukh $^{\mathrm{a}}$, Ngai Yin Yip ${ }^{\mathrm{a}, \mathrm{b}}$, Shihong Lin ${ }^{\mathrm{a}, \mathrm{c}}$, and Menachem Elimelech ${ }^{\mathrm{a}}$ \\ ${ }^{a}$ Department of Chemical and Environmental Engineering, Yale University \\ New Haven, Connecticut 06520-8286 \\ ${ }^{\mathrm{b}}$ Department of Earth and Environmental Engineering, Columbia University \\ New York, New York 10027 \\ ${ }^{\mathrm{c}}$ Department of Civil and Environmental Engineering, Vanderbilt University, \\ Nashville, Tennessee 37235-1831
}

*Corresponding author: Ngai Yin Yip; email: nyy2002@ columbia.edu 
In this study, we analyze the effects of membrane properties, namely water permeability, solute permeability, and structural parameter, on the overall performance of an FO membrane module to extract water from simulated seawater $(0.6 \mathrm{M} \mathrm{NaCl})$. By considering the thermodynamic limit of operation, we demonstrate that the maximum achievable water recovery is practically independent of membrane properties, and higher maximum water recovery is achievable with counter-current compared to co-current mode. Analysis of the module-scale model indicates that reducing the support layer structural parameter offers substantial reductions in the membrane area required to achieve a specified water recovery. For example, a $25 \%$ reduction of the structural parameter of a state-of-the-art thin-film composite (TFC) membrane (from 400 to 300 $\mu \mathrm{m}$ ) yields a sizable $20 \%$ reduction in membrane area. In contrast, quintupling the water permeability coefficient (from 2.0 to $10.0 \mathrm{~L} \mathrm{~m}^{-2} \mathrm{~h}^{-1} \mathrm{bar}^{-1}$ ) of a modern TFC membrane generates only a modest $10 \%$ saving in membrane area. In addition, because of the permeabilityselectivity trade-off that governs current polymeric membranes, doubling the water permeability coefficient would cause crippling $~ 7$-fold increases in forward and reverse solute permeation. This quantitative study models the potential performance of a module-scale FO desalination process and firmly highlights the need to prioritize the reduction of support layer mass transport resistances over water permeability increases in membrane development.

Keywords: Forward osmosis; Desalination; Membrane module analysis; Water recovery; Membrane area requirement

\section{Introduction}

Seawater desalination accounts for a rapidly increasing portion of global freshwater production [1,2]. Currently, reverse osmosis (RO) and multi-stage flash (MSF) separation are the most extensively employed desalination processes. Despite steady technological advances over the years, MSF remains highly energy intensive and RO still requires a substantial amount of highgrade energy in the form of electricity $[3,4]$. Recent studies have highlighted the inherent link between water and energy sustainability and emphasized the need to produce water in a manner 
which minimizes the use of high quality energy resources [4-6]. Emergent technologies such as forward osmosis (FO) and membrane distillation which can utilize low-grade waste energy are, therefore, of increasing importance in desalination [6-12]. FO desalination systems have a wide range of potential water production and purification applications, from hybrid FO-RO schemes for agricultural water that exploit the lower fouling propensity of FO [13], to shale gas water recycling systems that utilize the ability of FO to treat the highly saline waters produced during hydraulic fracturing $[14,15]$.

FO desalination comprises two stages: first, water is extracted from a saline feed stream into a draw solution and, second, the draw solute is separated from the diluted draw stream to recover the extracted water [1]. In the first stage, the saline feed solution is contacted with a high osmotic pressure draw solution in a membrane module. Driven by the chemical potential gradient, water permeates from the feed solution across the semipermeable membrane into the draw solution. In the second stage, product water is separated from the draw stream in a closed cycle, thus regenerating the initial draw solution. Various separation processes may be employed to recover water from the draw stream [8,15-17]. For example, thermolytic draw solutes, such as ammonia-carbon dioxide, may be stripped using low temperature distillation [8].

In order for FO desalination to be cost-effective, membranes need to achieve a high average water flux whilst maintaining an adequate rejection of salts and contaminants [1,7]. Currently, tailored thin-film composite (TFC) membranes, consisting of a thin polyamide active layer on a polysulfone-polyester fabric support layer, have demonstrated consistently high FO performance by providing the optimal combination of high water permeability, low solute permeability, and low support layer resistance to water transport [18-21]. Novel membranes, based on materials ranging from carbon nanotubes to graphene to aquaporin proteins, could potentially achieve greater water permeabilities and solute rejections, and are the subject of active research [22-26]. Innovative fabrication techniques are also being investigated in attempts to further lower the mass transport resistance of FO membrane support layers and enhance productivity [20,27-31]. 
Previous studies have investigated the trade-off between water and solute permeability of the membrane active layer [32,33] and the effect of morphology and microstructure on concentration polarization within the membrane support layer [28,34-38]. These studies concentrate on the theoretical and mechanistic understanding of the mass transport phenomena in FO and thus offer limited perspectives on overall FO system performance. At present, the advantages of improving membrane properties (water permeability and solute rejection of the active layer and mass transport resistance of the support layer) at module level have not been quantitatively analyzed. Module-scale modeling enables the determination of system-level performance parameters, including water recovery, solute leakage, and membrane area. In addition, incorporating the effects of local flow rate and concentration variations is imperative when assessing the impact of concentration polarization, which is exponentially dependent on the local water flux [39]. Therefore, module-scale FO analyses provide important practical insights on the economic viability of the process and can guide the design and implementation of FO desalination plants. Additionally, understanding the potential benefits of advances in membrane technology on FO module performance will be integral in steering future membrane development.

The objective of this study is to model a module-scale forward osmosis unit operation and examine the effects of key membrane transport parameters on performance. First, we consider the thermodynamic limits of water recovery for both co- and counter-current flow in an FO process. Second, we introduce mass transport across a differential FO membrane element and discuss the performance limiting phenomena. We then develop a module-scale process model to simulate an FO membrane unit and examine the contributions of active layer, support layer, and boundary layer mass transport resistances to the membrane area required to achieve a specified water recovery. Lastly, we investigate the effects of different operating conditions, specifically, feed and draw flow rates and concentrations, on the membrane area requirement. The findings of this study can serve to guide the design and development of forward osmosis systems by identifying the parameters that offer the largest potential improvements in process performance. 


\section{Upper Limit of Water Recovery}

An FO module consists of a low concentration feed stream and a high concentration draw stream separated by a semi-permeable membrane, commonly in a spiral-wound or tubular/hollow fiber configuration [40]. Driven by the osmotic pressure gradient, water permeates across the membrane from the feed stream to the draw stream [41]. Similarly, draw solutes are transported down a concentration gradient from the draw stream to the feed stream, while feed solutes permeate into the draw stream. The primary objective of an FO process is to recover water from the feed stream into the draw stream. Error! Reference source not found. shows schematics of two configurations of an FO process: co-current and counter-current.Error! Reference source not found.

Throughout this study, both the feed and draw solutes are taken to be $\mathrm{NaCl}$ and osmotic pressures are approximated using the van't Hoff equation, $\pi=v c R_{g} T$, where $v$ is the van't Hoff dissociation factor $(v=2$ for $\mathrm{NaCl}), R_{g}$ is the universal gas constant, $T$ is the absolute solution temperature, and $c$ is the molar salt concentration. The accuracy of the van't Hoff osmotic pressure approximation decreases substantially at solute concentrations above $1.5 \mathrm{M}$ [42]. However, in this study the feed and draw concentrations at the membrane-solution interface range from $0.6 \mathrm{M}$ to $1.4 \mathrm{M}$. In this region, errors in the van't Hoff approximation are under 3\% [43] (OLI Systems, Morris Plains, NJ). In addition, we neglect hydraulic pressure drop along the membrane module, as these are small in comparison to the osmotic pressure differences across the membrane. By applying these assumptions, two streams are in equilibrium with each other when they have the same concentration. Thin-film composite (TFC) polyamide membranes governed by solution-diffusion were utilized in the membrane modules for all model analysis. Other module-scale effects, such as configuration of the membrane elements, are not considered in this investigation.

We define the water recovery, $R$, and the feed flow rate fraction, $\phi$, in terms of the volumetric rate of water permeation across the membrane, $\Delta Q$, and the initial feed and draw flow rates, $Q_{F 0}$ and $Q_{D 0}$, respectively: 


$$
\begin{gathered}
R=\frac{\Delta Q}{Q_{F 0}} \\
\phi=\frac{Q_{F 0}}{Q_{F 0}+Q_{D 0}}
\end{gathered}
$$

Recovery, $R$, is the fraction of water extracted from the saline feed and is, thus, an important performance parameter in desalination processes.

Water flux across the membrane, $J_{w}$, is proportional to the osmotic pressure difference and can be expressed using the membrane water permeability coefficient, $A$, and feed and draw concentrations at the membrane-solution interface, $c_{F, m}$ and $c_{D, m}$, respectively [40]:

$$
J_{w}=A\left(\pi_{D, m}-\pi_{F, m}\right)=v A R_{g} T\left(c_{D, m}-c_{F, m}\right)
$$

Because concentrations $c_{F, m}$ and $c_{D, m}$ vary along the length of the membrane module, $J_{w}$ denotes the local water flux. Similarly, the local net solute flux, $J_{s}$, is expressed using the membrane solute permeability coefficient, $B[38,44]$ :

$$
J_{S}=B\left(c_{D, m}-c_{F, m}\right)
$$

Since both the draw and feed solutes are $\mathrm{NaCl}, J_{S}$ represents an aggregate of the reverse flux (RSF) of draw solute into the feed stream, $J_{S}^{R}$, and the forward flux of feed solute into the draw stream, $J_{S}^{F}$ (i.e., $J_{S}=J_{S}^{R}-J_{S}^{F}$ ) [44]. 
The integrals of $J_{w}$ and $J_{s}$ with respect to the membrane area over the entire module yield $\Delta Q$ and $\Delta N_{S}$, the net molar leakage rate of solute:

$$
\begin{gathered}
\Delta Q=\int_{0}^{A_{m}} J_{w} d A_{m}^{\prime}=v A R_{g} T \int_{0}^{A_{m}}\left(c_{D, m}-c_{F, m}\right) d A_{m}^{\prime} \\
\Delta N_{s}=\int_{0}^{A_{m}} J_{s} d A_{m}^{\prime}=B \int_{0}^{A_{m}}\left(c_{D, m}-c_{F, m}\right) d A_{m}^{\prime}
\end{gathered}
$$

where $A_{m}$ is the area of the membrane surface. From Eqs. (5) and (6) we note that the molar leakage rate of solute is directly proportional to the rate of water permeation across a membrane module [45]. Using this proportionality relation with Eq. (1) we can express $\Delta N_{S}$ in terms of the water recovery:

$$
\frac{\Delta N_{s}}{R Q_{F 0}}=\frac{B}{v A R_{g} T}
$$

To determine the theoretical upper bound of water recovery in FO, we consider the limiting scenario in which the exiting feed and draw streams are in thermodynamic equilibrium (i.e. equal osmotic pressures or, in our case, equal salt concentrations) with adjacent streams, which may be entering (counter-current, Error! Reference source not found.b) or exiting (cocurrent, Error! Reference source not found.a) the module [41]. This modeling condition implicitly assumes a module with sufficient membrane area across which water and solute mass transfer can proceed to equilibrium. We define solute leakage (SL) as the cumulative permeation of solute across the entire membrane module. Using Eq. (7) to account for net SL (i.e. reverse minus forward solute permeation), we determine the dependence of the maximum water recovery on $\phi$ and $c_{D 0}$ for co- and counter-current flow. 


\subsection{Co-Current Flow}

In co-current flow, the feed and draw streams exit the module on the same end. By setting the exit concentrations of both streams to be equal and performing a mass balance on water and salt, the maximum attainable water recovery can be expressed in terms of the initial concentrations, feed flow rate fraction, and membrane water and solute permeability coefficients with Eqs. (1), (2), and (7):

$$
R_{\rightrightarrows}=\frac{(1-\phi)\left(c_{D 0}-c_{F 0}\right)}{\phi c_{F 0}+(1-\phi) c_{D 0}+\frac{B}{v A R_{g} T}}
$$

\subsection{Counter-Current Flow}

In counter-current flow, the feed and draw streams run in an anti-parallel manner, with the feed exit being adjacent to the draw entrance and vice versa. This configuration yields two possible equilibrium conditions - equal concentrations at the feed or draw inlet of the membrane module. We define $\phi^{*}$ as the critical feed flow rate fraction that satisfies both conditions simultaneously and $R^{*}$ as the corresponding critical recovery. Evaluating this critical flow rate fraction using water and solute mass balance yields:

$$
\phi^{*}=\frac{c_{D 0}+\frac{B}{v A R_{g} T}}{c_{D 0}+c_{F 0}+\frac{2 B}{v A R_{g} T}}
$$

When the feed flow rate fraction is smaller than the critical value, i.e. $\phi<\phi^{*}$, the concentration of the feed stream increases along the module to equal the draw inlet concentration, while the concentration of the draw stream does not fully decrease to that of the feed inlet. This is defined as the feed limiting regime (FLR) and the maximum attainable recovery, $R_{\rightleftarrows, F L R}$, is equal to the critical recovery, $R^{*}$, which is given by: 


$$
R^{*}=R_{\rightleftarrows, F L R}=\frac{c_{D 0}-c_{F 0}}{c_{D 0}+\frac{B}{v A R_{g} T}}
$$

Conversely, the draw limiting regime (DLR) occurs when the feed flow rate fraction is greater than the critical value, i.e. $\phi>\phi^{*}$. In this case, the concentration of the draw stream decreases to equal the feed inlet concentration, while the concentration of the feed stream does not increase to that of the draw inlet. The maximum attainable recovery in DLR is:

$$
R_{\rightleftarrows, D L R}=\frac{(1-\phi)\left(c_{D 0}-c_{F 0}\right)}{\phi\left(c_{F 0}+\frac{B}{v A R_{g} T}\right)}
$$

\subsection{Effect of Solute Permeation on Water Recovery}

The transport of water and salts through current polymeric membranes is described by the solution-diffusion mechanism [46]. Consequently, polymeric membranes are constrained by an intrinsic trade-off between permeability and selectivity. In this study, the permeabilityselectivity correlation for TFC polyamide membranes [32,33] is used to calculate the dependence of the solute permeability coefficient, $B$, on the water permeability coefficient, $A$ :

$$
B=\gamma A^{3}
$$

where constant $\gamma=0.0133 \mathrm{~L}^{-2} \mathrm{~m}^{4} \mathrm{~h}^{2} \mathrm{bar}^{3}$ is experimentally determined in our previous study [33].

Note that the denominators of Eqs. (8), (10), and (11) contain the term $B /\left(v A R_{g} T\right)$, which has dimensions of concentration. This term represents the reduction in osmotic driving force due to net SL to the feed side, which consequently leads to a decrease in water recovery. Currently, thin-film composite polyamide membranes offer the best combination of high water permeability and high solute rejection [19]. Using the representative concentrations and typical TFC membrane properties presented in Table 1, the term is calculated to be two orders of magnitude smaller than the feed inlet concentration ( $0.6 \mathrm{M} \mathrm{NaCl}$ to simulate seawater). 

small when high-selectivity polyamide membranes are employed. Nonetheless, because the leakage of draw solute will incur material replenishment cost, added steps to counter the accumulation of feed solute in the draw side, and possibly the additional treatment of feed stream, SL is still anticipated to be an important phenomenon in actual operation and is discussed later in Section 4.4.

Table 1Error! Reference source not found. shows the dependence of the maximum attainable water recovery on the feed flow rate fraction for co- and counter-current flow (black and red lines, respectively) as detailed in Eqs. (8), (10), and (11). It is immediately evident that running an FO system in counter-current mode allows a higher water recovery relative to running in cocurrent mode, for $0<\phi<1$. The superior performance of counter-current operation is the consequence of maintaining a higher average driving force (i.e. osmotic pressure difference) along the module. It is for this reason that separation processes are commonly operated in counter-current mode [47]. Because of the clear advantage in achievable recovery, only countercurrent configurations are modeled in subsequent sections.Error! Reference source not found.

For counter-current flow, there are two distinct regimes on either side of the critical feed flow rate fraction $\left(\phi^{*}=0.83\right.$ for $c_{F 0}=0.6 \mathrm{M}$ and $\left.c_{D 0}=3.0 \mathrm{M}\right)$. In the FLR (i.e. $\phi<\phi^{*}$ ), water recovery remains constant at its maximum value, $R_{\rightleftarrows, F L R}=0.80$ for $c_{D 0}=3.0 \mathrm{M}$ (i.e. Eq. (10) is independent of $\phi$ ). This is because the draw stream has sufficient capacity to continue extracting water from the feed stream until the feed concentration increases to that of the draw inlet. In contrast, water recovery in the DLR (i.e. $\phi>\phi^{*}$ ) drops rapidly as the feed flow rate fraction is increased, as reflected by the $(1-\phi) / \phi$ term in Eq. (11). In this region, the flow rate of the draw stream is insufficient and its concentration decreases to that of the feed inlet.

Changing the draw inlet concentration affects both the maximum recovery possible and the critical feed flow rate fraction, Eqs. (9), (10), and (11). Decreasing the draw inlet concentration in counter-current flow, from $c_{D 0}=3.0$ to $1.2 \mathrm{M}$, decreases both the maximum attainable water recovery, $R_{\rightleftarrows, F L R}=0.8$ to 0.5 , and the critical feed flow rate fraction, $\phi^{*}=0.83$ to 0.67 (Error! Reference source not found., red lines). Therefore, employing a higher draw 


\section{Element-Scale Analysis: Performance Limiting Phenomena in FO}

The driving force for water flux in FO is the osmotic pressure difference across the active layer of the membrane, $\Delta \pi_{m}=\pi_{D, m}-\pi_{F, m}$, Eq. (3). Due to concentration polarization (CP) and RSF, the osmotic pressure difference across the active layer, $\Delta \pi_{m}$, is lower than the osmotic pressure difference between the bulk feed and draw streams, $\Delta \pi_{b}=\pi_{D, b}-\pi_{F, b}$. The effects of $\mathrm{CP}$ and RSF on the steady-state water and salt fluxes are quantified by modeling the convective and diffusive transport of species across the membrane support, active layer, and the adjacent boundary later [48]. A schematic of the osmotic pressure profile is shown in Error! Reference source not found..Error! Reference source not found.

Concentrative external concentration polarization (ECP) occurs on the feed-side, at the solution-membrane interface, and is characterized by a mass transfer coefficient, $k_{F}$ [48]. Its reciprocal, $1 / k_{F}$, represents the resistance of the diffusive boundary layer to solute transport. The feed-side mass transfer coefficient is dependent on the hydrodynamics of the flow in the feed channel and varies with the feed-side flow rate $[49,50]$. The relationship between the mass transfer coefficient and flow rate is typically expressed via semi-empirical Sherwood-ReynoldsSchmidt number correlations [49,51]. In this study a constant mass transfer coefficient is used rather than assuming a particular channel and spacer geometry and using the corresponding Sherwood-Reynolds-Schmidt correlation. The mass transfer coefficient can be improved by increasing the cross-flow velocity or enhancing spacer design in the feed channel and thus induce greater mixing near the membrane surface [50,52].

On the draw side, dilutive internal concentration polarization (ICP) occurs inside the support layer. ICP is characterized by the structural parameter, $S$, of the support layer and the diffusivity, $\mathcal{D}$, of the draw solute in water. The structural parameter, $S=t_{S} \tau / \varepsilon$, is a membrane property which incorporates the thickness, $t_{S}$, tortuosity, $\tau$, and porosity, $\varepsilon$, of the support layer and represents an effective diffusive length scale within the support [53]. A thick, low porosity, 
and highly tortuous support layer hinders the diffusion of solutes from the bulk solution to the active-support layer interface, thus diminishing the accessible draw solution osmotic driving force. Dilutive ECP also occurs on the draw-side of the membrane; however, its impact is small relative to ICP and its effects are assimilated into the structural parameter [48].

RSF lowers the osmotic driving force by increasing the solute concentration, and hence osmotic pressure, on the feed-side of the active layer whilst reducing $c_{D, m}[45,54,55]$. The magnitude of the RSF is characterized by the solute permeability coefficient, $B$, of the membrane. The extent to which RSF reduces the water flux depends on the degree of feed-side ECP. If $k_{F}$ is high (i.e., extensive mixing), leaked draw solute will readily diffuse from the active layer-feed solution interface into the bulk feed stream and the detrimental effects of RSF will be slight. If there is little mixing and $k_{F}$ is low, RSF will noticeably exacerbate the feed-side ECP concentration gradient causing a substantial reduction in the local water flux.

Using steady-state film theory, the local water flux across a differential membrane element incorporating the effects of ECP, ICP, and net solute flux is given by [48]:

$$
J_{w}=\frac{A\left[\pi_{D, b} \exp \left(-\frac{J_{W} S}{\mathcal{D}}\right)-\pi_{F, b} \exp \left(\frac{J_{w}}{k_{F}}\right)\right]}{1+\frac{B}{J_{w}}\left[\exp \left(\frac{J_{w}}{k_{F}}\right)-\exp \left(-\frac{J_{W} S}{\mathcal{D}}\right)\right]}
$$

The terms $\exp \left(-J_{W} S / \mathcal{D}\right)$ and $\exp \left(J_{W} / k_{F}\right)$ are the CP moduli of dilutive ICP and concentrative ECP, respectively. The term $\left(B / J_{W}\right)\left[\exp \left(J_{W} / k_{F}\right)-\exp \left(-J_{W} S / \mathcal{D}\right)\right]$ in the denominator of Eq. (13) represents the reduction in water flux due to the net effect of solute permeation. Similarly, the net solute flux, $J_{S}=J_{S}^{R}-J_{S}^{F}$, is given by $[45,48]$ :

$$
J_{S}=\frac{B\left[c_{D, b} \exp \left(-\frac{J_{w} S}{\mathcal{D}}\right)-c_{F, b} \exp \left(\frac{J_{w}}{k_{F}}\right)\right]}{1+\frac{B}{J_{w}}\left[\exp \left(\frac{J_{W}}{k_{F}}\right)-\exp \left(-\frac{J_{w} S}{\mathcal{D}}\right)\right]}
$$




\section{Module-Scale Analysis}

Membrane area is a key parameter in the design of engineered osmotic systems [9]. Membrane modules are approximately $30 \%$ of the capital expenditure and $10 \%$ of the operational expenditure of current reverse osmosis desalination systems [56]. Consequently, developing membrane modules that can achieve a large water recovery using a smaller membrane area is vital to the cost effectiveness of FO desalination. In addition, the areal footprint of a desalination plant is advantageously reduced with a smaller membrane area requirement. In this section, we examine the effects of various membrane transport properties and operating parameters on the minimum membrane area required to obtain a specified water recovery.

\subsection{Concentration and Flow Rate Profiles in an Counter-Current FO Module}

In Section 2, we analyzed mass transfer across an infinite membrane area to calculate the maximum water recovery achievable in an FO module. In Section 3, we considered mass transfer across an infinitesimal membrane area whilst accounting for the transport-limiting phenomena. In this section, we examine mass transfer across a finite membrane area to analyze the impact of various membrane transport parameters on the membrane area required to achieve a specified water recovery, to simulate the full-scale unit operation of FO desalination. This approach simultaneously accounts for the variations in osmotic driving force along an FO module and the mass transport limitations across an FO membrane. In these analyses, the feed and draw concentrations are $0.6 \mathrm{M}$ and $3.0 \mathrm{M} \mathrm{NaCl}$, respectively (Table 1).

Two governing equations determine the flow rates and concentrations in a countercurrent FO module: a volumetric balance on water, Eq. (15), and a molar balance on salt, Eq. (16):

$$
\begin{gathered}
\frac{d Q_{F}}{d A_{m}^{\prime}}=\frac{d Q_{D}}{d A_{m}^{\prime}}=-J_{w} \\
\frac{d\left(Q_{F} c_{F}\right)}{d A_{m}^{\prime}}=\frac{d\left(Q_{D} c_{D}\right)}{d A_{m}^{\prime}}=J_{S}
\end{gathered}
$$


where $A_{m}^{\prime}$ denotes membrane area in the direction of the feed stream.

Module-scale flow rate, concentration, and flux profiles are numerically determined by solving the mass conservation equations, Eqs. (15) and (16), in conjunction with the water and salt flux equations, Eqs. (13) and (14). Numerical solutions are obtained by dividing the membrane area into finite elements across which the implicit water and solute flux equations are solved. An iterative approach is employed using Python (Python Software Foundation, DE) to solve counter-current systems by estimating and then adjusting the exiting draw stream flow rate (refer to Supplementary Material for details on modeling methodology). Error! Reference source not found. shows the flow rate fractions (red) and concentrations (black) of the feed stream (dashed, entering from the left) and draw stream (solid, entering from the right) along a representative counter-current FO module. The membrane and transport properties are as described in Table 1. The local water flux (blue) along the membrane module is also shown. With initial $\phi=0.8$ and $c_{D, m}=3.0 \mathrm{M} \mathrm{NaCl}$, a recovery of $R=0.35$ was achieved with $0.025 \mathrm{~m}^{2}$ of membrane area per $1.00 \mathrm{Lh}^{-1}$ of feed flow $(0.6 \mathrm{M} \mathrm{NaCl}$ to simulate seawater).Error! Reference source not found.

\subsection{Effect of Membrane Properties on Membrane Area Requirement}

Error! Reference source not found. shows a contour plot of the membrane area required to obtain a recovery of 0.5 , as a function of membrane properties. The feed flow rate fraction is $0.8, c_{D, m}=3.0 \mathrm{M}$, and $c_{F, m}=0.6 \mathrm{M}$. The membrane water permeability coefficient, $A$, and structural parameter, $S$, are represented on the bottom horizontal axis and vertical axis, respectively, while the solute permeability coefficient, $B$, calculated using the permeabilityselectivity trade-off, Eq. (12), is indicated on the top horizontal axis. Membrane area, $A_{m}$, is normalized by the inlet feed flow rate, $Q_{F 0}$, to ensure that the trends observed are scalable. The black lines denote the values of the water permeability coefficient, $A$, that yield the minimum membrane area (solid line) and $110 \%$ of the minimum membrane area (dashed line) area, given a specified structural parameter, $S$. Error! Reference source not found. 
For typical TFC membrane parameters, Error! Reference source not found. shows that lowering the structural parameter yields a much greater decrease in normalized membrane area than increasing the water permeability coefficient. For instance, doubling the water permeability coefficient (from 2.0 to $4.0 \mathrm{~L} \mathrm{~m}^{-2} \mathrm{~h}^{-1} \mathrm{bar}^{-1}$ ) gives a $5.4 \%$ decrease in the membrane area, while quintupling $A$ from 2.0 to $10.0 \mathrm{~L} \mathrm{~m}^{-2} \mathrm{~h}^{-1} \mathrm{bar}^{-1}$ generates only a modest $10 \%$ membrane area saving. On the other hand, a 25\% reduction of the structural parameter of a state-of-the-art TFC membrane (from 400 to $300 \mu \mathrm{m}$ ) yields a sizable $20 \%$ reduction in membrane area, and halving $S$ from 400 to $200 \mu$ m yields a substantially larger decrease of $40 \%$. In addition, the black curves in Error! Reference source not found. demonstrate that although a high water permeability coefficient is required to minimize the membrane area at a specified structural parameter, e.g. $A=6.16 \mathrm{~L} \mathrm{~m}^{-2} \mathrm{~h}^{-1}$ bar $^{-1}$ for $S=400 \mu \mathrm{m}, 110 \%$ of this minimum area can be realized with a far lower permeability coefficient, $A=1.59 \mathrm{~L} \mathrm{~m}^{-2} \mathrm{~h}^{-1} \mathrm{bar}^{-1}$ in this example. This trend is prevalent across the entire range of structural parameters displayed.

Due to the intrinsic permeability-selectivity trade-off, increasing the water permeability coefficient rapidly increases the salt permeability coefficient, $B \propto A^{3}$, as shown in Eq. (12). As the water and salt permeability is increased for a specific structural parameter in Error! Reference source not found. (i.e., going horizontally left to right), the normalized membrane area decreases, plateaus and then increases gently, rather than precipitously. This reinforces the earlier discussion in Section 2 that reverse solute permeation does not significantly impact water permeation (and, hence, membrane area) when the feed is relatively saline, $c_{F, b}=0.6 \mathrm{M}$. For saline feed solutions, the solute concentration at the feed-membrane interface is not significantly elevated by RSF. However, as the draw solute leaks across to the feed side and accumulates along the membrane module, the bulk osmotic driving force is lowered. The gradual increase in membrane area requirement as the permeability coefficients are increased beyond the solid black line is chiefly attributed to the increased build-up of leaked draw solute in the feed side and its depletion from the draw side.

To assess how the relative impact of internal and external concentration varies with water recovery, simulations were performed assuming ECP and/or ICP were absent (i.e., $k_{F} \rightarrow \infty, S=0$ ). Error! Reference source not found. shows the minimum normalized 
membrane area as a function of water recovery. The red slice represents the hypothetical minimum area requirement for a membrane module without ECP or ICP, the green slice represents the additional area required due to ECP (without ICP), and the blue slice represents the additional area required due to ICP. Water and solute permeability coefficients as well as the support layer structural parameter are as presented in

Table 1.Error! Reference source not found.Error! Reference source not found. clearly demonstrates the dominant contribution of ICP to the normalized membrane area across a range of water recoveries. Over the range of $R$ examined, ECP doubled the hypothetical membrane area requirement, while ICP caused a drastic tenfold increase of $A_{m}$. Thus, Error! Reference source not found. illustrates that ICP in the support layer provides the principal resistance to mass transfer in typical FO operation, irrespective of the required water recovery.

\subsection{Membrane Area Requirement can be Most Effectively Reduced by Improving Structural Parameter}

Enhancing the hydrodynamic mixing on the feed side, for example by increasing the cross-flow velocity or improving spacer design, can suppress the detrimental effects of ECP [13,17] and lower the required membrane area. Recent studies have also suggested that creating micrometerscale channels on the membrane surface may reduce ECP by increasing surface turbulence [58]. However, these beneficial membrane area reductions will likely be achieved at the expense of additional pumping energy cost.

Increasing the membrane water permeability coefficient can reduce the membrane area requirement, but will cause a quadratic increase in the molar rate of net SL, i.e. $\Delta N_{s} \propto A^{2}$, Eq. (7). Therefore in the earlier example for Error! Reference source not found., the 5.4\% saving in membrane area made possible by a 2-fold increase in the membrane permeability coefficient (from 2.0 to $4.0 \mathrm{~L} \mathrm{~m}^{-2} \mathrm{~h}^{-1} \mathrm{bar}^{-1}$ ) will come at the expense of a 4-fold increase in the rate of net SL. The disadvantageous outcomes of a higher solute leakage on module-scale operation is further examined in the following section. 
Rather than targeting more permeable, but inevitably less selective, membranes, the development efforts of FO membranes should strategically focus on lessening the adverse effects of ICP. Both Error! Reference source not found. and Error! Reference source not found. strongly indicate that reducing the structural parameter offers the greatest improvements in process performance, comprehensively eclipsing benefits gained from increasing the water permeability coefficient or feed-side mass transfer coefficient. Novel membranes (employing materials such as aligned nanotubes, graphene, and aquaporin proteins) promising higher water permeabilities will likely only offer limited membrane area savings in FO desalination unless the support layer structural parameter is also radically improved. Reductions in the structural parameter may be achieved by reducing the thickness and tortuosity, increasing the porosity and hydrophilicity of the membrane support [5,21,59], or developing novel support layers [28,31].

\subsection{Solute Leakage Across More Permeable, Less Selective Membranes Outweighs Benefits of Membrane Area Reduction}

The rate of solute leakage discussed thus far is the cumulative molar rate, $\Delta N_{s}$, encompassing forward and reverse SL. Forward SL permits salts and contaminants from the feed stream to advance into the second stage of FO desalination. Additional separations or purges will then be required to regulate the accumulation of feed solutes in the closed-loop second desalination stage. Similarly, high reverse SL rates could severely limit process viability, particularly if the draw solute is expensive or hazardous $[45,60]$. Post-treatment of the feed stream will be required to recover or remove the draw solute, thus introducing additional energetic and economic costs.

For an FO module, operating at a water recovery of $R=0.5$, with membrane properties as described in Table 1, doubling the water permeability coefficient, $A$, from 2.00 to $4.00 \mathrm{~L} \mathrm{~m}^{-2} \mathrm{~h}^{-1}$ bar $^{-1}$ triggers a 7.2-fold increase in forward SL (from 7.21 to 51.7 moles of solute leaked per cubic meter of water recovered) and a 6.8-fold increase in reverse SL (from 8.29 to $56.0 \mathrm{~mol} \mathrm{~m}^{-3}$ ). This considerable deleterious increase in forward and reverse SL is accompanied only by a mere $5.4 \%$ saving in membrane area provided by the enhanced water permeability. In comparison, halving the structural parameter, S, from 400 to $200 \mu \mathrm{m}$ offers a $40.4 \%$ saving in membrane area in addition to $35.6 \%$ and $31.7 \%$ decreases in forward and 
reverse SL, respectively. Thus, for typical polymeric membranes with $A$ values in the region of $2.0 \mathrm{~L} \mathrm{~m}^{-2} \mathrm{~h}^{-1}$ bar $^{-1}$ (Table 1), increasing water permeability will likely have a detrimental impact on overall FO performance due to the greater trade-off in reduced membrane selectivity. Here, innovative membranes (e.g., aligned nanotubes, graphene, and aquaporin proteins) that potentially offer better permeability-selectivity over the conventional polyamide thin-film composite can reduce the forward and reverse solute leakage while maintaining water recovery.

\subsection{Lower Feed Flow Rate Fraction and Higher Inlet Draw Concentration Reduce Minimum Area Requirement}

To study the effect of operating conditions on membrane area requirement, the module-scale analysis was carried out for water recovery of 0.5 (typical recovery rate of seawater RO desalination plants [61,62]). Error! Reference source not found. illustrates the dependence of the minimum membrane area required for $R=0.5$ on two operational parameters: feed flow rate fraction and inlet draw concentration. The feed solution is $0.6 \mathrm{M} \mathrm{NaCl}$ to simulate seawater. Reducing the feed flow rate fraction and increasing the inlet draw concentration both reduce the minimum required membrane area. This result is consistent with the earlier findings on the maximum achievable recovery of the thermodynamic analysis in Section 2.Error! Reference source not found.

Raising the inlet draw concentration increases the osmotic driving force along the FO module, thereby raising the average water flux. Lowering the feed flow rate fraction decreases the decline in the osmotic driving force along the FO module, again raising the average water flux. Because a greater water flux is maintained along the membrane module, less membrane area is required to achieve the target water recovery. Hence, operating FO desalination with a relatively larger flow rate of highly concentrated draw solution is favorable for reducing the membrane area requirement. However, these methods of reducing the membrane area may be limited in their scope and effectiveness depending on the nature of the draw solution and the overall desalination process. For example, increases in the draw solution concentration may be restricted by the solubility of the draw solute [60]. 


\section{Concluding Remarks}

In this study, we model and evaluate the impact of membrane properties on the performance of FO desalination at the module-scale. The model incorporated the permeability-selectivity tradeoff and quantified the thermodynamic limits of water recovery for co- and counter-current flow. We showed that solute permeability does not have a significant effect on water recovery and higher recoveries are achievable in counter- than co-current configuration. We developed a module-scale FO model and demonstrated that internal concentration polarization constituted the largest resistance to water permeation. The findings of this study strongly suggest that advances aimed at lowering the structural parameter will offer the most substantial improvements in membrane performance. Our modeling asserts that further increasing the water permeability coefficient of current FO membranes will not yield significant reduction in membrane area. In addition, for current polymeric membranes governed by solution-diffusion, there is an optimal permeability-selectivity that balances the membrane area requirement with the operational concerns of forward and reverse SL. Willful pursuit of greater membrane permeability inevitably lowers the selectivity at greater rates and the detrimental effects will comprehensively outweigh the marginal reductions in membrane area. 


\section{References}

[1] M. Elimelech, W.A.W. Phillip, The future of seawater desalination: energy, technology, and the environment, Science. 333 (2011) 712-717.

[2] Q. Schiermeier, Water: purification with a pinch of salt., Nature News. 452 (2008) 260-1.

[3] G. Raluy, L. Serra, J. Uche, Life cycle assessment of MSF, MED and RO desalination technologies, Energy. 31 (2006) 2361-2372.

[4] R. Semiat, Energy Issues in Desalination Processes, Environmental Science \& Technology. 42 (2008) 8193-8201.

[5] L.A. Hoover, W.A. Phillip, A. Tiraferri, N.Y. Yip, M. Elimelech, Forward with osmosis: Emerging applications for greater sustainability, Environmental Science \& Technology. 45 (2011) 9824-9830.

[6] R.L. McGinnis, M. Elimelech, Global Challenges in Energy and Water Supply: The Promise of Engineered Osmosis, Environmental Science \& Technology. 42 (2008) 8625-8629.

[7] D.L. Shaffer, J.R. Werber, H. Jaramillo, S. Lin, M. Elimelech, Forward osmosis: Where are we now?, Desalination. (2014).

[8] J.R. McCutcheon, R.L. McGinnis, M. Elimelech, A novel ammonia-carbon dioxide forward (direct) osmosis desalination process, Desalination. 174 (2005) 1-11.

[9] M. a Shannon, P.W. Bohn, M. Elimelech, J.G. Georgiadis, B.J. Marinas, a M. Mayes, Science and technology for water purification in the coming decadesie, Nature. 452 (2008) 301-310.

[10] Z. Liu, H. Bai, J. Lee, D.D. Sun, A low-energy forward osmosis process to produce drinking water, Energy \& Environmental Science. 4 (2011) 2582-2585.

[11] L. Martínez-Díez, F.J. Florido-Díaz, Desalination of brines by membrane distillation, Desalination. 137 (2001) 267-273.

[12] M.P. Godino, L. Peña, C. Rincón, J.I. Mengual, Water production from brines by membrane distillation, Desalination. 108 (1997) 91-97.

[13] D.L. Shaffer, N.Y. Yip, J. Gilron, M. Elimelech, Seawater desalination for agriculture by integrated forward and reverse osmosis: Improved product water quality for potentially less energy, Journal of Membrane Science. 415-416 (2012) 1-8.

[14] D.L. Shaffer, L.H. Arias Chavez, M. Ben-Sasson, S. Romero-Vargas Castrillón, N.Y. Yip, M. Elimelech, Desalination and reuse of high-salinity shale gas produced water: drivers, technologies, and future directions., Environmental Science \& Technology. 47 (2013) 9569-9583. 
[15] R.L. McGinnis, N.T. Hancock, M.S. Nowosielski-Slepowron, G.D. McGurgan, Pilot demonstration of the $\mathrm{NH} 3 / \mathrm{CO} 2$ forward osmosis desalination process on high salinity brines, Desalination. 312 (2013) 67-74.

[16] M.L. Stone, C. Rae, F.F. Stewart, A.D. Wilson, Switchable polarity solvents as draw solutes for forward osmosis, Desalination. 312 (2013) 124-129.

[17] M.M. Ling, K.Y. Wang, T.-S. Chung, Highly Water-Soluble Magnetic Nanoparticles as Novel Draw Solutes in Forward Osmosis for Water Reuse, Industrial \& Engineering Chemistry Research. 49 (2010) 5869-5876.

[18] N. Widjojo, T.-S. Chung, M. Weber, C. Maletzko, V. Warzelhan, The role of sulphonated polymer and macrovoid-free structure in the support layer for thin-film composite (TFC) forward osmosis (FO) membranes, Journal of Membrane Science. 383 (2011) 214-223.

[19] N. Yip, A. Tiraferri, W.A. Phillip, J.D. Schiffman, M. Elimelech, High performance thin-film composite forward osmosis membrane, Environmental Science \& Technology. 44 (2010) 38123818 .

[20] A. Tiraferri, Y. Kang, E.P. Giannelis, M. Elimelech, Superhydrophilic thin-film composite forward osmosis membranes for organic fouling control: fouling behavior and antifouling mechanisms., Environmental Science \& Technology. 46 (2012) 11135-11144.

[21] A. Tiraferri, N.Y. Yip, W.A. Phillip, J.D. Schiffman, M. Elimelech, Relating performance of thinfilm composite forward osmosis membranes to support layer formation and structure, Journal of Membrane Science. 367 (2011) 340-352.

[22] B. Corry, Designing carbon nanotube membranes for efficient water desalination., The Journal of Physical Chemistry. 112 (2008) 1427-34.

[23] Y. Jia, H. Li, M. Wang, L. Wu, Y. Hu, Carbon nanotube: Possible candidate for forward osmosis, Separation and Purification Technology. 75 (2010) 55-60.

[24] X. Peng, J. Jin, Y. Nakamura, T. Ohno, I. Ichinose, Ultrafast permeation of water through proteinbased membranes., Nature Nanotechnology. 4 (2009) 353-7.

[25] E.N. Wang, R. Karnik, Water desalination: Graphene cleans up water., Nature Nanotechnology. 7 (2012) 552-554.

[26] M. Kumar, M. Grzelakowski, J. Zilles, M. Clark, W. Meier, Highly permeable polymeric membranes based on the incorporation of the functional water channel protein Aquaporin Z., Proceedings of the National Academy of Sciences of the United States of America. 104 (2007) 20719-24.

[27] P. Sukitpaneenit, T.-S. Chung, High performance thin-film composite forward osmosis hollow fiber membranes with macrovoid-free and highly porous structure for sustainable water production., Environmental Science \& Technology. 46 (2012) 7358-7365. 
[28] L.A. Hoover, J.D. Schiffman, M. Elimelech, Nanofibers in thin-film composite membrane support layers: Enabling expanded application of forward and pressure retarded osmosis, Desalination. 308 (2013) 73-81.

[29] K.Y. Wang, R.C. Ong, T.-S. Chung, Double-Skinned Forward Osmosis Membranes for Reducing Internal Concentration Polarization within the Porous Sublayer, Industrial \& Engineering Chemistry Research. 49 (2010) 4824-4831.

[30] S. You, C. Tang, C. Yu, X. Wang, J. Zhang, J. Han, et al., Forward osmosis with a novel thin-film inorganic membrane., Environmental Science \& Technology. 47 (2013) 8733-8742.

[31] X. Song, Z. Liu, D.D. Sun, Nano gives the answer: breaking the bottleneck of internal concentration polarization with a nanofiber composite forward osmosis membrane for a high water production rate., Advanced Materials. 23 (2011) 3256-3260.

[32] G.M. Geise, H.B. Park, A.C. Sagle, B.D. Freeman, J.E. McGrath, Water permeability and water/salt selectivity tradeoff in polymers for desalination, Journal of Membrane Science. 369 (2011) 130-138.

[33] N. Yip, M. Elimelech, Performance limiting effects in power generation from salinity gradients by pressure retarded osmosis, Environmental Science \& Technology. 45 (2011) 10273-10282.

[34] H.Y. Ng, W. Tang, W.S. Wong, Performance of Forward (Direct) Osmosis Process: Membrane Structure and Transport Phenomenon, Environmental Science \& Technology. 40 (2006) 24082413.

[35] J.R. McCutcheon, M. Elimelech, Influence of concentrative and dilutive internal concentration polarization on flux behavior in forward osmosis, Journal of Membrane Science. 284 (2006) 237247.

[36] J. McCutcheon, M. Elimelech, Modeling water flux in forward osmosis: implications for improved membrane design, AIChE Journal. 53 (2007) 1736-1744.

[37] J.R. McCutcheon, R.L. McGinnis, M. Elimelech, Desalination by ammonia-carbon dioxide forward osmosis: Influence of draw and feed solution concentrations on process performance, Journal of Membrane Science. 278 (2006) 114-123.

[38] S. Loeb, L. Titelman, E. Korngold, J. Freiman, Effect of porous support fabric on osmosis through a Loeb-Sourirajan type asymmetric membrane, Journal of Membrane Science. 129 (1997) 243249.

[39] M. Mulder, J. Caro, Basic Principles of Membrane Technology, Kluwer Academic Pub, 1998.

[40] T.Y. Cath, A.E. Childress, M. Elimelech, Forward osmosis: Principles, applications, and recent developments, Journal of Membrane Science. 281 (2006) 70-87. 
[41] S. Phuntsho, S. Hong, M. Elimelech, H.K. Shon, Osmotic equilibrium in the forward osmosis process: Modelling, experiments and implications for process performance, Journal of Membrane Science. 453 (2014) 240-252.

[42] C.H. Tan, H.Y. Ng, Modified models to predict flux behavior in forward osmosis in consideration of external and internal concentration polarizations, Journal of Membrane Science. 324 (2008) 209-219.

[43] A.P. Straub, N.Y. Yip, M. Elimelech, Raising the Bar: Increased Hydraulic Pressure Allows Unprecedented High Power Densities in Pressure-Retarded Osmosis, Environmental Science \& Technology Letters. 1 (2014) 55-59.

[44] N.T. Hancock, W.A. Phillip, M. Elimelech, T.Y. Cath, Bidirectional permeation of electrolytes in osmotically driven membrane processes., Environmental Science \& Technology. 45 (2011) 10642-10651.

[45] W.A. Phillip, J.S. Yong, M. Elimelech, Reverse draw solute permeation in forward osmosis: modeling and experiments., Environmental Science \& Technology. 44 (2010) 5170-6.

[46] D. Paul, Reformulation of the solution-diffusion theory of reverse osmosis, Journal of Membrane Science. 241 (2004) 371-386.

[47] R.H. Perry, D.W. Green, Perry’s Chemical Engineers' Handbook, McGraw-Hill, 2008.

[48] A. Tiraferri, N.Y. Yip, A.P. Straub, S. Romero-Vargas Castrillon, M. Elimelech, A method for the simultaneous determination of transport and structural parameters of forward osmosis membranes, Journal of Membrane Science. 444 (2013) 523-538.

[49] E.M. V. Hoek, A.S. Kim, M. Elimelech, Influence of Crossflow Membrane Filter Geometry and Shear Rate on Colloidal Fouling in Reverse Osmosis and Nanofiltration Separations, Environmental Engineering Science. 19 (2002) 357-372.

[50] V. Gekas, B. Hallström, Mass transfer in the membrane concentration polarization layer under turbulent cross flow, Journal of Membrane Science. 30 (1987) 153-170.

[51] Z.V.P. Murthy, S.K. Gupta, Estimation of mass transfer coefficient using a combined nonlinear membrane transport and film theory model, Desalination. 109 (1997) 39-49.

[52] G. Guillen, E.M.V. Hoek, Modeling the impacts of feed spacer geometry on reverse osmosis and nanofiltration processes, Chemical Engineering Journal. 149 (2009) 221-231.

[53] K.L. Lee, R.W. Baker, H.K. Lonsdale, Membranes for power generation by pressure-retarded osmosis, Journal of Membrane Science. 8 (1981) 141-171.

[54] C. Suh, S. Lee, Modeling reverse draw solute flux in forward osmosis with external concentration polarization in both sides of the draw and feed solution, Journal of Membrane Science. 427 (2013) 365-374. 
[55] N.T. Hancock, T.Y. Cath, Solute Coupled Diffusion in Osmotically Driven Membrane Processes, Environmental Science \& Technology. 43 (2009) 6769-6775.

[56] American Membrane Technology Association, Seawater Desalination Costs, http://www.amtaorg.com/wp-content/uploads/6_MembraneDesalinationCosts.pdf, 2012.

[57] P. Bacchin, D. Si-Hassen, V. Starov, M.. Clifton, P. Aimar, A unifying model for concentration polarization, gel-layer formation and particle deposition in cross-flow membrane filtration of colloidal suspensions, Chemical Engineering Science. 57 (2002) 77-91.

[58] S.H. Maruf, A.R. Greenberg, J. Pellegrino, Y. Ding, Fabrication and characterization of a surfacepatterned thin film composite membrane, Journal of Membrane Science. 452 (2014) 11-19.

[59] N.-N. Bui, M.L. Lind, E.M.V. Hoek, J.R. McCutcheon, Electrospun nanofiber supported thin film composite membranes for engineered osmosis, Journal of Membrane Science. 385-386 (2011) 1019.

[60] A. Achilli, T.Y. Cath, A.E. Childress, Selection of inorganic-based draw solutions for forward osmosis applications, Journal of Membrane Science. 364 (2010) 233-241.

[61] L.F. Greenlee, D.F. Lawler, B.D. Freeman, B. Marrot, P. Moulin, Reverse osmosis desalination: water sources, technology, and today's challenges., Water Research. 43 (2009) 2317-48.

[62] M. Wilf, K. Klinko, Optimization of seawater RO systems design, Desalination. 138 (2001) 299306.

[63] Tampa Bay Water, Tampa Bay Seawater Desalination, http://www.tampabaywater.org/tampa-bayseawater-desalination-plant.aspx, 2010.

[64] S. Lee, C. Boo, M. Elimelech, S. Hong, Comparison of fouling behavior in forward osmosis (FO) and reverse osmosis (RO), Journal of Membrane Science. 365 (2010) 34-39.

[65] J. Wei, C. Qiu, C.Y. Tang, R. Wang, A.G. Fane, Synthesis and characterization of flat-sheet thin film composite forward osmosis membranes, Journal of Membrane Science. 372 (2011) 292-302.

[66] T.Y. Cath, M. Elimelech, J.R. McCutcheon, R.L. McGinnis, A. Achilli, D. Anastasio, et al., Standard Methodology for Evaluating Membrane Performance in Osmotically Driven Membrane Processes, Desalination. 312 (2013) 31-38. 


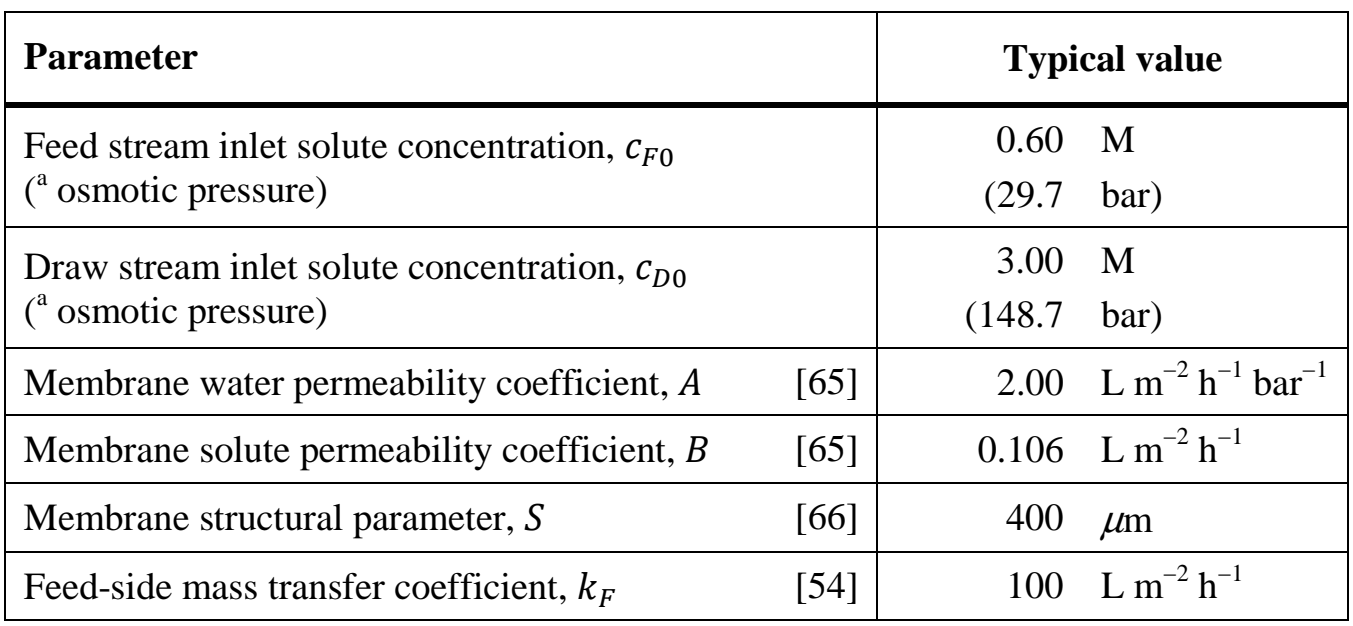

a The osmotic pressures are approximated using the van't Hoff equation. 
a)

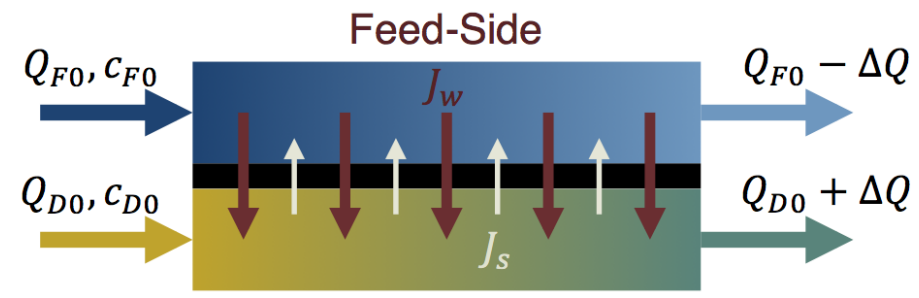

Draw-Side

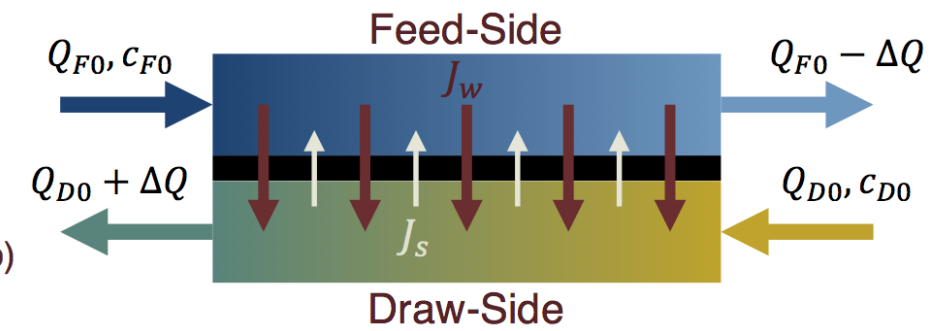

Fig. 1. Schematic of a FO module operating in (a) co-current and (b) counter-current mode. Water permeates from the feed-side to the draw-side with a local flux of $J_{w}$. The permeation flow rate, $\Delta Q$, is the integral of $J_{w}$ across the membrane area of the entire module. Draw solute leaks from the draw-side to the feed-side with a molar flux of $J_{S}^{R}$. Feed solute permeates from the feed-side to the draw-side with a molar flux of $J_{S}^{F}$. The net solute flux comprising $J_{S}^{R}$ and $J_{S}^{F}$ from the draw-side to the feed-side is $J_{S}$. The volumetric flow rate and molar concentration of the streams are denoted by $Q$ and $c$, respectively. 


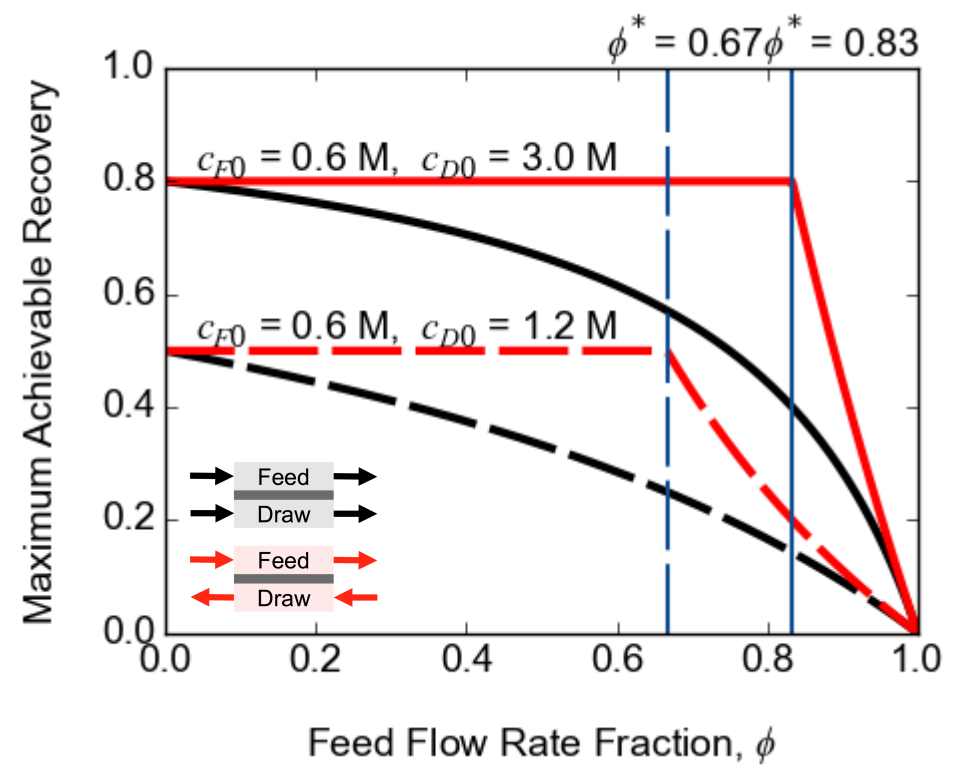

Fig. 2: Maximum achievable water recovery as a function of feed flow rate fraction. Solid and dashed lines represent a draw solute concentration of $c_{D 0}=3.0 \mathrm{M}$ and $1.2 \mathrm{M} \mathrm{NaCl}$, respectively, while the black and red lines denote co- and counter-current flow configurations, respectively. All curves are plotted for a feed concentration of $c_{F 0}=0.6 \mathrm{M} \mathrm{NaCl}$, simulating seawater. Membrane parameters presented in Table 1 were used. 


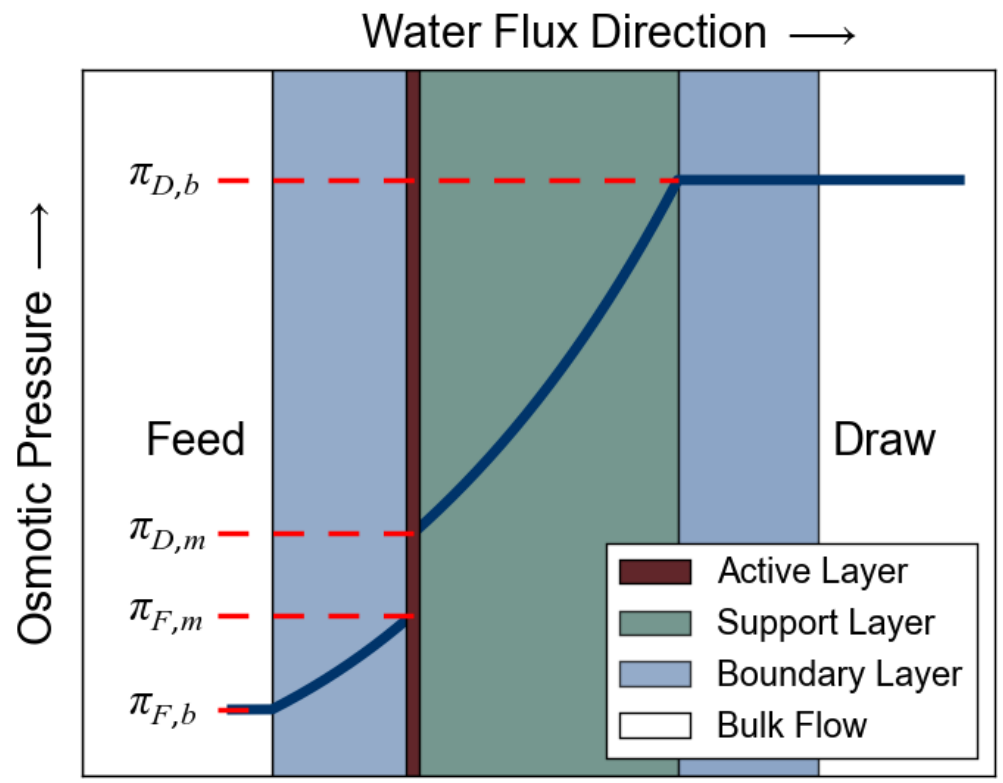

Fig. 3: Schematic of the osmotic pressure profile across the membrane from the bulk feed stream to the bulk draw stream. The boundary layer at the feed-membrane interface is modeled using film theory. 


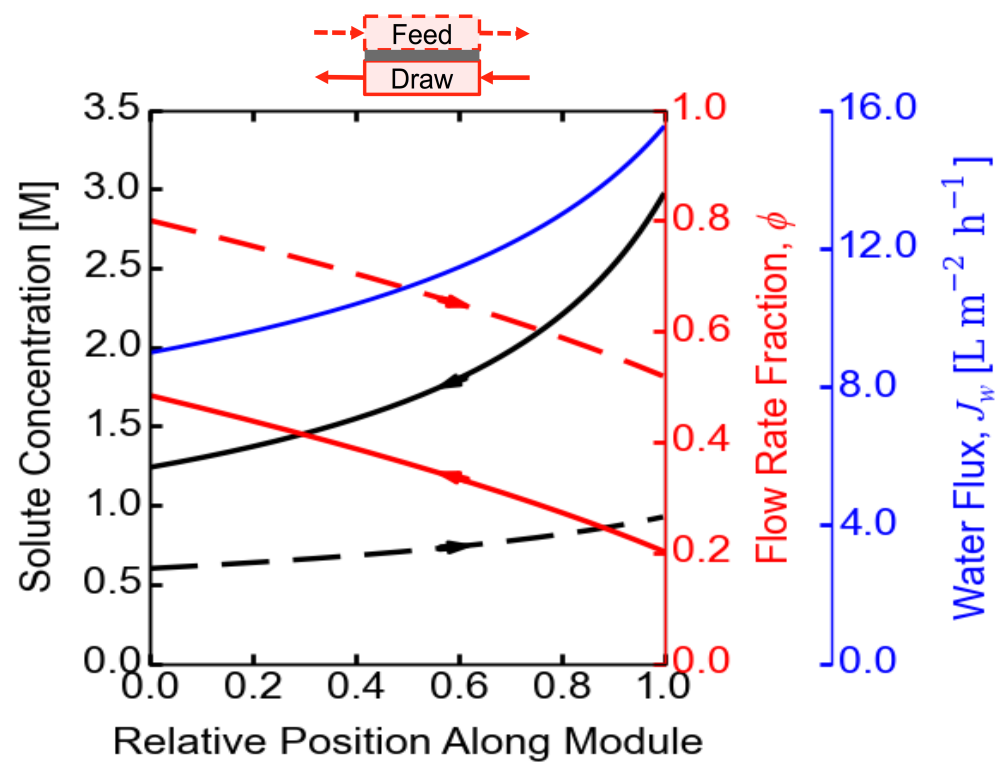

Fig. 4: Representative flow rate fraction (red), solute concentration (black), and water flux (blue) profiles along a FO module operating in counter-current mode. The feed stream (dashed) enters from the left (position $=0$ ) and flows rightward while the draw stream (solid) enters from the right (position $=1$ ) and flows leftward. The normalized membrane area is $A_{m} / Q_{F 0}=$ $0.025\left(\mathrm{~L} \mathrm{~m}^{-2} \mathrm{~h}^{-1}\right)^{-1}$ and the initial feed flow rate fraction is $\phi=0.8$. Recovery of $R=0.35$ is achieved in this illustrative example. Other membrane and transport properties are as presented in Table 1. 


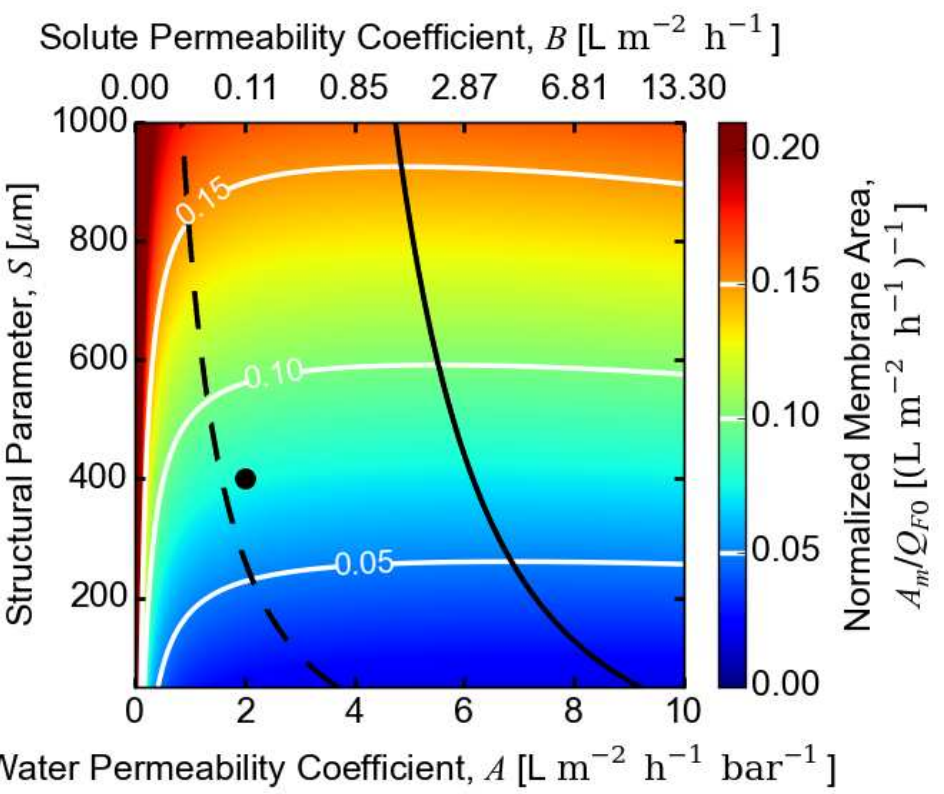

Fig. 5: Contour plot of the normalized membrane area, $A_{m} / Q_{F 0}$, required to obtain a recovery of $R=0.5$ with a feed flow rate fraction of $\phi=0.8$, as a function of membrane water permeability coefficient, $A$, and structural parameter, $S$. The feed-side mass transfer coefficient is $k_{F}=$ $100 \mathrm{~L} \mathrm{~m}^{-2} \mathrm{~h}^{-1}$, and the solute permeability, $B$, is calculated using the permeability-selectivity correlation, Eq. 12, and indicated on the top horizontal axis. The solid black line represents the loci of $A$ values that minimize $A_{m} / Q_{F 0}$ for a specified $S$ value, while the dotted black line denote $A$ values that yield $110 \%$ of the minimum $A_{m} / Q_{F 0}$. The black dot represents the properties of the membrane presented in Table 1: $A=2.00 \mathrm{~L} \mathrm{~m}^{-2} \mathrm{~h}^{-1} \mathrm{bar}^{-1}$ and $S=400 \mu \mathrm{m}$. 


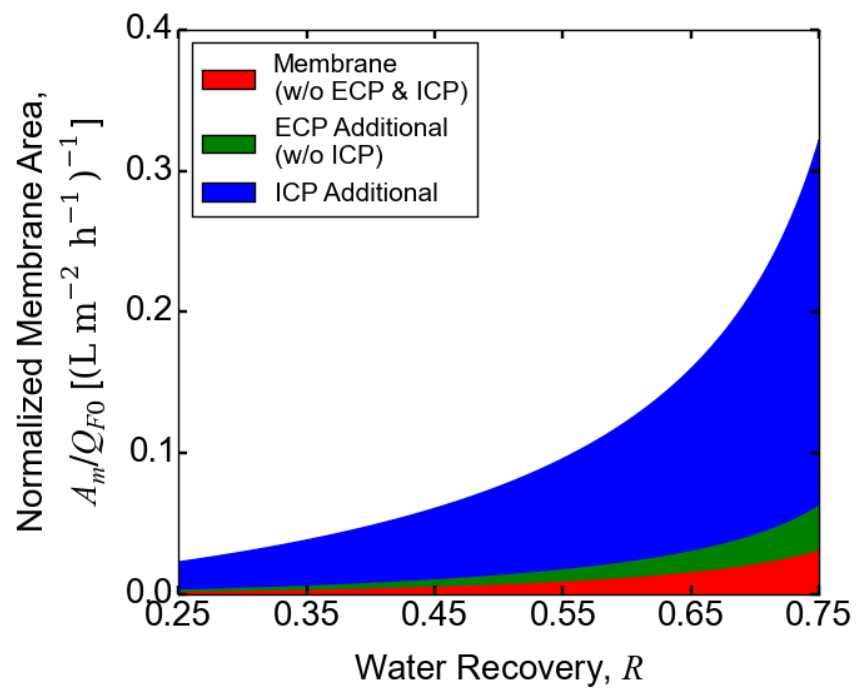

Fig. 6: Dependence of the normalized minimum membrane area on water recovery. The membrane area has been normalized using the inlet feed stream flow rate. The red baseline slice represents the area for a membrane module with no $\mathrm{CP}$. The green and blue slices represent the additional membrane area required due to the effects of ECP and ICP respectively. A feed flow rate fraction of $\phi=0.8$ is used along with the membrane and transport properties presented in Table 1. 


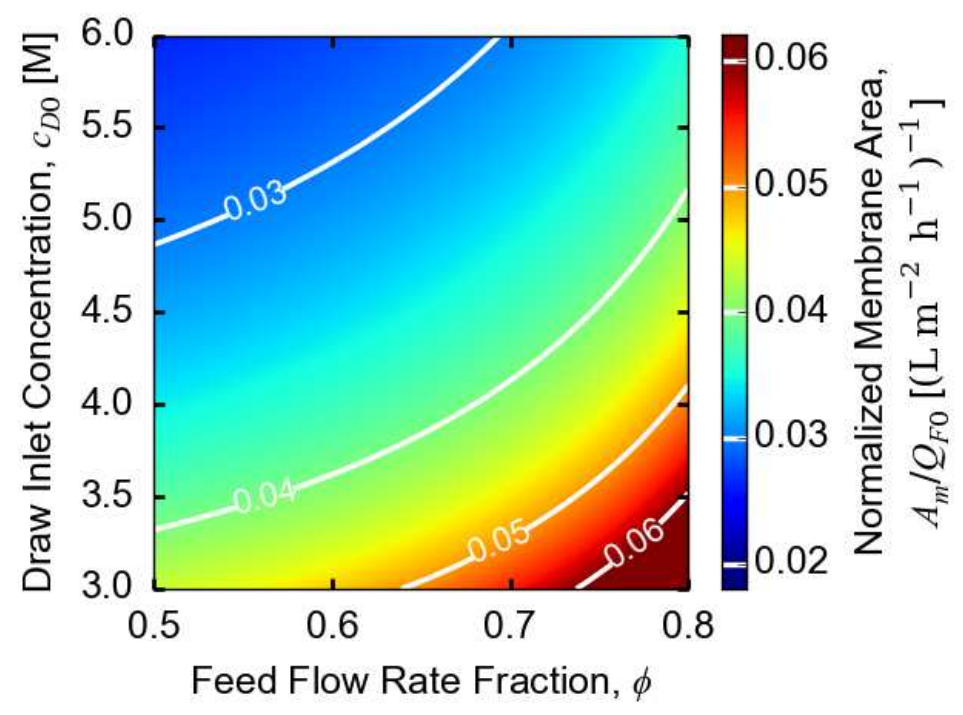

Fig. 7: Contour plot of the minimum membrane area required to achieve a recovery of $R=0.5$ as a function of feed flow rate fraction and draw inlet concentration (horizontal and vertical axis, respectively). The membrane area is normalized by the inlet feed flow rate. The feed solution is $0.6 \mathrm{M} \mathrm{NaCl}$ and membrane and transport properties are as presented in Table 1. 